Article

\title{
A New Reproducing Kernel Approach for Nonlinear Fractional Three-Point Boundary Value Problems
}

\author{
Mehmet Giyas Sakar *(D) and Onur Saldır \\ Faculty of Sciences, Department of Mathematics, Van Yuzuncu Yil University, 65080 Van, Turkey; \\ onursaldir@yyu.edu.tr \\ * Correspondence: giyassakar@yyu.edu.tr; Tel.: +90-(432)-2251701-27895
}

Received: 30 June 2020; Accepted: 20 November 2020; Published: 24 November 2020

check for updates

\begin{abstract}
In this article, a new reproducing kernel approach is developed for obtaining a numerical solution of multi-order fractional nonlinear three-point boundary value problems. This approach is based on a reproducing kernel, which is constructed by shifted Legendre polynomials (L-RKM). In the considered problem, fractional derivatives with respect to $\alpha$ and $\beta$ are defined in the Caputo sense. This method has been applied to some examples that have exact solutions. In order to show the robustness of the proposed method, some examples are solved and numerical results are given in tabulated forms.
\end{abstract}

Keywords: shifted Legendre polynomials; reproducing kernel method; variable coefficient; Caputo derivative; three-point boundary value problem

\section{Introduction}

In this paper, a new iterative reproducing kernel approach will be constructed for obtaining the numerical solution of a multi-order fractional nonlinear three-point boundary value problem as follows:

$$
\begin{gathered}
a_{2}(\xi){ }^{c} D^{\alpha} z(\xi)+a_{1}(\xi){ }^{c} D^{\beta} z(\xi)+a_{0}(\xi) z(\xi)=g\left(\xi, z(\xi), z^{\prime}(\xi)\right) \\
0 \leq \xi \leq 1,1<\alpha \leq 2,0<\beta \leq 1 .
\end{gathered}
$$

with the following boundary conditions,

$$
z(0)=\gamma_{0}, z(\theta)=\gamma_{1}, z(1)=\gamma_{2}, 0<\theta<1 .
$$

Here, $a_{0}(\cdot), a_{1}(\cdot), a_{2}(\cdot) \in C^{2}(0,1)$ and $g\left(\cdot, z(\cdot), z^{\prime}(\cdot)\right) \in L_{\rho}^{2}[0,1]$ are sufficiently smooth functions, $\rho$ is a weighted function and it will be taken as $\rho=1$ for Legendre polynomials. Fractional derivatives are also taken in the Caputo sense. Without loss of generality, we pay regards to $z(0)=0, z(\theta)=0$ and $z(1)=0$. Because $z(0)=\gamma_{0}, z(\theta)=\gamma_{1}$ and $z(1)=\gamma_{2}$, boundary conditions can be easily reduced to $z(0)=0$, $z(\theta)=0$ and $z(1)=0$.

Nonlinear fractional multi-point boundary value problems appear in a different area of applied mathematics and physics ([1-7] and references therein). Many important phenomena have been concerned in engineering and applied science, such as dynamical systems, fluid mechanics, control theory, oil industries, and heat conduction, and can be well-turned by fractional differential equations [8-10]. 
Some applications, qualitative behaviors of solutions and numerical methods to find approximate solutions have been investigated for differential equations with fractional order in [11-14].

More specfically, it is not easy to directly get exact solutions to most differential equations with fractional order. Hence, numerical techniques are largely utilized. Actually, in recent times, many efficient and convenient methods have been developed, such as the finite difference method [15], finite element method [16], homotopy perturbation method [17], Haar wavelet methods [18], collocation methods [19], homotopy analysis method [20], differential transform method [21], variational iteration method [22], reproducing kernel space method $[23,24]$ and so on [25-32].

In 1908, Zaremba firstly introduced the reproducing kernel concept [33]. His researches regarded boundary value problems, which include the Dirichlet condition. The reproducing kernel method (RKM) produces a solution in convergent series form for many differential, partial and integro-differential equations. For more information, we refer to [34,35]. Recently, the RKM has been applied for a different type of problem. For example, fractional order nonlocal boundary value problems [36], Riccati differential equations [37], forced Duffing equations with a nonlocal boundary conditions [38], Burgers' equation with a fractional order Caputo derivative [39], time-fractional Kawahara equation [40], fractional order Boussinesq equation [41], nonlinear fractional Volterra integro-differential equations [42].

The Legendre reproducing kernel method is proposed for the fractional two-point boundary value problem of Bratu type equations [43]. The main motivation of this paper is to extend the Legendre reproducing kernel approach for solving multi-order fractional nonlinear three-point boundary value problems with a Caputo derivative.

The remainder part of the paper is prepared as follows: some fundamental definitions of fractional calculus and the theory of reproducing kernel with Legendre basis functions are given in Section 2. The structure of the solution with a Legendre reproducing kernel is demonstrated in Section 3. In order to show the effectiveness of the proposed method, some numerical findings are reported in Section 4. Finally, the last section contains some conclusions.

\section{Preliminaries}

In this section, several significant concepts, definitions, theorems, and properties that will be used in this research are provided.

Definition $1([8,12,13])$. Let $z(\xi) \in C[0,1]$ and $\xi \in[0,1]$. Then, the $\alpha$ order Riemann-Liouville fractional integral operator is given as:

$$
J^{\alpha} z(\xi)=\frac{1}{\Gamma(\alpha)} \int_{0}^{\xi}(\xi-s)^{\alpha-1} z(s) d s,
$$

here $\Gamma($.$) is a Gamma function, \alpha \geq 0$ and $\xi>0$.

Definition $2([8,12,13])$. Let $z(\xi) \in A C[0,1]$ and $\xi \in[0,1]$. Then, the $\alpha$ order Caputo differential operator is given as:

$$
{ }^{c} D^{\alpha} z(\xi)=\frac{1}{\Gamma(a-\alpha)} \int_{0}^{\xi} \frac{z^{(a)}(s)}{(\xi-s)^{\alpha+1-a}} d s, a-1<\alpha \leq a, a \in \mathbb{N} \text { and } \xi>0 .
$$


Definition 3 ([26,43]). In order to a construct polynomial type reproducing kernel, the first kind of shifted Legendre polynomials are defined over the interval $[0,1]$. For obtaining these polynomials, the following iterative formula can be given:

$$
\begin{aligned}
P_{0}(\xi) & =1 \\
P_{1}(\xi) & =2 \xi-1, \\
& \vdots \\
(n+1) P_{n+1}(\xi) & =(2 n+1)(2 \xi-1) P_{n}(\xi)-n P_{n-1}(\xi), n=1,2, \ldots
\end{aligned}
$$

The orthogonality requirement is

$$
\left\langle P_{n}, P_{m}\right\rangle=\int_{0}^{1} \rho_{[0,1]}(\xi) P_{n}(\xi) P_{m}(\xi) d \xi= \begin{cases}0, & n \neq m \\ 1, & n=m=0 \\ \frac{1}{2 n+1}, & n=m \neq 0\end{cases}
$$

here, the weighted function is taken as,

$$
\rho_{[0,1]}(\xi)=1 .
$$

Legendre basis functions can be established so that this basis function system satisfies the homogeneous boundary conditions as:

$$
z(0)=0 \text { and } z(1)=0 .
$$

Equation (5) has an advantageous feature for solving boundary value problems. Therefore, these basis functions for $j \geq 2$ can be defined as;

$$
\phi_{j}(\xi)= \begin{cases}P_{j}(\xi)-P_{0}(\xi), & j \text { is even }, \\ P_{j}(\xi)-P_{1}(\xi), & j \text { is odd. }\end{cases}
$$

such that this system satisfies the conditions

$$
\phi_{j}(0)=\phi_{j}(1)=0 .
$$

It is worth noting that the basis functions given in Equation (6) are a complete system. For more information about orthogonal polynomials, please see [44-46].

Definition 4. Let $\Psi \neq \varnothing$, and $\mathbb{H}$ with its inner product $\langle\cdot, \cdot\rangle_{\mathbb{H}}$ be a Hilbert space of real-valued functions on $\Psi$. Then, the reproducing kernel of $\mathbb{H}$ is $R: \Psi \times \Psi \rightarrow \mathbb{R}$ iff

1. $R(\cdot, \xi) \in \mathbb{H}, \forall \xi \in \Psi$

2. $\langle\phi(\cdot), R(\cdot, \xi)\rangle_{\mathbb{H}}=\phi(\xi), \forall \phi \in \mathbb{H}, \forall \xi \in \Psi$.

The last condition is known as a reproducing property. Especially, for any $x, \xi \in \Psi$,

$$
R(x, \xi)=\langle R(\cdot, x), R(\cdot, \xi)\rangle_{\mathbb{H} \cdot}
$$


If a Hilbert space satisfies the above two conditions then it is called a reproducing kernel Hilbert space. The uniqueness of the reproducing kernel with respect to the inner product can be shown by the use of the Riesz representation theorem [47].

Theorem 1. Let $\left\{e_{j}\right\}_{j=1}^{n}$ be an orthonormal basis of n-dimensional Hilbert space $\mathbb{H}$, then

$$
R(x, \xi)=R_{x}(\xi)=\sum_{j=1}^{n} \bar{e}_{j}(x) e_{j}(\xi)
$$

is a reproducing kernel of $\mathbb{H}[34,35]$.

Definition 5. Let $\Omega_{\rho}^{m}[0,1]$ polynomials space be the pre-Hilbert space over $[0,1]$ with real coefficients and its degree $\leq m$ and inner product as:

$$
\langle z, v\rangle_{\Omega_{\rho}^{m}}=\int_{0}^{1} \rho_{[0,1]}(\xi) z(\xi) v(\xi) d \xi, \forall z, v \in \Omega_{\rho}^{m}[0,1],
$$

with $\rho_{[0,1]}$ described by Equation (4), and the norm

$$
\|z\|_{\Omega_{\rho}^{m}}=\sqrt{\langle z, z\rangle}_{\Omega_{\rho}^{m}}, \forall z \in \Omega_{\rho}^{m}[0,1] .
$$

With the aid of definiton of $L^{2}$ Hilbert space, $L_{\rho}^{2}[0,1]=\left\{\left.g\left|\int_{0}^{1} \rho_{[0,1]}(\xi)\right| g(\xi)\right|^{2} d \xi<\infty\right\}$ for any fixed $m$, $\Omega_{\rho}^{m}[0,1]$ is a subspace of $L_{\rho}^{2}[0,1]$ and $\forall z, v \in \Omega_{\rho}^{m}[0,1],\langle z, v\rangle_{\Omega_{\rho}^{m}}=\langle z, v\rangle_{L_{\rho}^{2}}$.

Theorem 2 ([43]). $\Omega_{\rho}^{m}[0,1]$ Hilbert space is a reproducing kernel space.

Definition 6. Let

$$
{ }^{0} \Omega_{\rho}^{m}[0,1]=\left\{z \mid z \in \Omega_{\rho}^{m}[0,1], z(0)=z(1)=0\right\} .
$$

One can easily demonstrate that ${ }^{0} \Omega_{\rho}^{m}[0,1]$ is a reproducing kernel space using Equation (6). From Theorem 1, the kernel function $R_{x}^{m}(\xi)$ of ${ }^{0} \Omega_{\rho}^{m}[0,1]$ can be written as

$$
R_{x}^{m}(\xi)=\sum_{j=2}^{m} h_{j}(\xi) h_{j}(x)
$$

here, $h_{j}(\xi)$ is complete system, which is easily obtained from the basis functions in Equation (6) with the help of the Gram-Schmidt orthonormalization process. Equation (11) is very useful for implementation. In other words, $R_{x}^{m}(\xi)$ and $\Omega_{\rho}^{m}[0,1]$ can be readily updated and re-calculated by increasing $m$.

\section{Main Results}

In this section, some important results related to the reproducing kernel method with shifted Legendre polynomials are presented. In the first subsection, the generation of reproducing kernel that satisfies three-point boundary value problems is presented. In the second subsection, the representation of a solution is given in ${ }^{\theta} \Omega_{\rho}^{m}[0,1]$. Then, we will construct an iterative process for a nonlinear problem in the third subsection. 


\subsection{Generation of Reproducing Kernel for Three-Point Boundary Value Problems}

In this subsection, we shall generate a reproducing kernel Hilbert space ${ }^{\theta} \Omega_{\rho}^{m}[0,1]$ in which every functions satisfies $z(0)=0, z(\theta)=0$ and $z(1)=0$ for $\theta \in(0,1)$. Namely, ${ }^{\theta} \Omega_{\rho}^{m}[0,1]$ is defined as ${ }^{\theta} \Omega_{\rho}^{m}[0,1]=\left\{z \mid z \in \Omega_{\rho}^{m}[0,1], z(0)=z(\theta)=z(1)=0\right\}$.

Obviously, ${ }^{\theta} \Omega_{\rho}^{m}[0,1]$ reproducing kernel space is a closed subspace of ${ }^{0} \Omega_{\rho}^{m}[0,1]$. The reproducing kernel of ${ }^{\theta} \Omega_{\rho}^{m}[0,1]$ can be given with the following theorem.

Theorem 3. The reproducing kernel of ${ }^{\theta} \Omega_{\rho}^{m}[0,1]$ is ${ }^{\theta} R_{x}^{m}(\xi)$ :

$$
{ }^{\theta} R_{x}^{m}(\xi)=R_{x}^{m}(\xi)-\frac{R_{x}^{m}(\theta) R_{\theta}^{m}(\xi)}{R_{\theta}^{m}(\theta)}
$$

Proof. Frankly, not all elements of ${ }^{0} \Omega_{\rho}^{m}[0,1]$ vanish at $\theta$. This shows that $R_{\theta}^{m}(\theta) \neq 0$. Hence, it can be easily seen that ${ }^{\theta} R_{x}^{m}(\theta)={ }^{\theta} R_{\theta}^{m}(\xi)=0$ and therefore ${ }^{\theta} R_{x}^{m}(\xi) \in{ }^{\theta} \Omega_{\rho}^{m}[0,1]$. For $\forall z(x) \in{ }^{\theta} \Omega_{\rho}^{m}[0,1]$, clearly, $z(\theta)=0$, it follows that

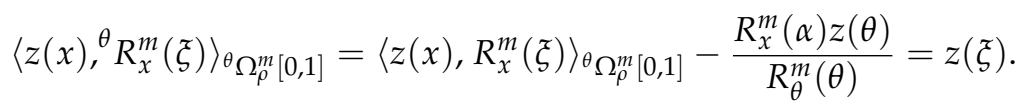

Namely, ${ }^{\theta} R_{x}^{m}(\xi)$ is a reproducing kernel of ${ }^{\theta} \Omega_{\rho}^{m}[0,1]$. This completes the proof.

\subsection{Representation of Solution in ${ }^{\theta} \Omega_{\rho}^{m}[0,1]$ Hilbert Space}

In this subsection, the reproducing kernel method with Legendre polynomials is established for obtaining a numerical solution of a three-point boundary value problem. For Equations (1) and (2), the approximate solution shall be constructed in ${ }^{\theta} \Omega_{\rho}^{m}[0,1]$. Firstly, we will define the linear operator $L$ as follows,

$$
L:{ }^{\theta} \Omega_{\rho}^{m}[0,1] \rightarrow L_{\rho}^{2}[0,1]
$$

such that

$$
L z(\xi):=a_{2}(\xi){ }^{c} D^{\alpha} z(\xi)+a_{1}(\xi){ }^{c} D^{\beta} z(\xi)+a_{0}(\xi) z(\xi) .
$$

Therefore, Equations (1) and (2) can be stated as follows

$$
\left\{\begin{array}{l}
L z=g\left(\xi, z(\xi), z^{\prime}(\xi)\right) \\
z(0)=z(\theta)=z(1)=0 .
\end{array}\right.
$$

It can easily be shown that the linear operator $L$ is bounded. We will obtain the representation solution of Equation (13) in the ${ }^{\theta} \Omega_{\rho}^{m}[0,1]$ space. Let ${ }^{\theta} R_{x}^{m}(\xi)$ be the polynomial form of the reproducing kernel in ${ }^{\theta} \Omega_{\rho}^{m}[0,1]$ space.

Theorem 4 ([43]). Let $\left\{\xi_{j}\right\}_{j=0}^{m-2}$ be any $(m-1)$ distinct points in open interval $(0,1)$ for Equations (1) and (2), then $\psi_{j}^{m}(\xi)=L^{* \theta} R_{\xi_{j}}^{m}(\xi)=\left.L_{x}{ }^{\theta} R_{x}^{m}(\xi)\right|_{x=\xi_{j}}$ for $m \geq 2$.

Theorem 5 ([43]). Let $\left\{\xi_{j}\right\}_{j=0}^{m-2}$ be any $(m-1)$ distinct points in open interval $(0,1)$ for $m \geq 2$, then $\left\{\psi_{j}^{m}\right\}_{j=0}^{m-2}$ is complete in ${ }^{\theta} \Omega_{\rho}^{m}[0,1]$. 
Theorem 5 indicates that in the Legendre reproducing kernel approach, using finite distinct points is enough. However, in the traditional reproducing kernel method needs a dense sequence on the interval. So, this new approach varies from the traditional method in $[27,36-39,42]$.

The orthonormal system $\left\{\bar{\psi}_{j}^{m}\right\}_{j=0}^{m-2}$ of ${ }^{\theta} \Omega_{\rho}^{m}[0,1]$ can be derived with the help of the Gram-Schmidt orthogonalization process using $\left\{\psi_{j}^{m}\right\}_{j=0}^{m-2}$,

$$
\bar{\psi}_{j}^{m}(\xi)=\sum_{k=0}^{j} \beta_{j k}^{m} \psi_{k}^{m}(\xi)
$$

here, $\beta_{j k}^{m}$ shows the coefficients of orthogonalization.

Theorem 6. Suppose that $z_{m}$ is the exact solution of Equations (1) and (2) and $\left\{\xi_{j}\right\}_{j=0}^{m-2}$ shows any $(m-1)$ distinct points in open interval $(0,1)$ for $m \geq 2$; in that case, the approximate solution $z_{m}(\xi)$ can be expressed as

$$
z_{m}(\xi)=\sum_{j=0}^{m-2} \sum_{k=0}^{j} \beta_{j k}^{m} g\left(\xi_{k}, z_{m}\left(\xi_{k}\right), z_{m}^{\prime}\left(\xi_{k}\right)\right) \bar{\psi}_{j}^{m}(\xi) .
$$

Proof. Since $z_{m} \in{ }^{\theta} \Omega_{\rho}^{m}[0,1]$, from Theorem 5 , the following equality can be written

$$
z_{m}(\xi)=\sum_{i=0}^{m-2}\left\langle z_{m}(\xi), \bar{\psi}_{j}^{m}(\xi)\right\rangle_{\theta} \Omega_{\rho}^{m} \bar{\psi}_{j}^{m}(\xi)
$$

On the other part, using Theorem 4 and Equation (14), we obtain $z_{m}(\xi)$, which is the precise solution of Equation (10) in ${ }^{\theta} \Omega_{\rho}^{m}[0,1]$ as,

$$
\begin{aligned}
z_{m}(\xi) & =\sum_{j=0}^{m-2}\left\langle z_{m}(\xi), \bar{\psi}_{j}^{m}(\xi)\right\rangle_{{ }_{\theta} \Omega_{\rho}^{m}} \bar{\psi}_{j}^{m}(\xi)=\sum_{j=0}^{m-2}\left\langle z_{m}(\xi), \sum_{k=0}^{j} \beta_{j k}^{m} \psi_{k}^{m}(\xi)\right\rangle_{{ }_{\theta} \Omega_{\rho}^{m}} \bar{\psi}_{j}^{m}(\xi) \\
& =\sum_{j=0}^{m-2} \sum_{k=0}^{j} \beta_{j k}^{m}\left\langle z_{m}(\xi), \psi_{k}^{m}(\xi)\right\rangle_{{ } \Omega_{\rho}^{m}} \bar{\psi}_{j}^{m}(\xi)=\sum_{j=0}^{m-2} \sum_{k=0}^{j} \beta_{j k}^{m}\left\langle z_{m}(\xi), L^{* \theta} R_{\xi_{k}}^{m}(\xi)\right\rangle_{{ }_{\theta} \Omega_{\rho}^{m}} \bar{\psi}_{j}^{m}(\xi) \\
& =\sum_{j=0}^{m-2} \sum_{k=0}^{j} \beta_{j k}^{m}\left\langle L z_{m}(\xi),{ }^{\theta} R_{\xi_{k}}^{m}(\xi)\right\rangle_{L_{\rho}^{2}} \bar{\psi}_{j}^{m}(\xi)=\sum_{j=0}^{m-2} \sum_{k=0}^{j} \beta_{j k}^{m}\left\langle g\left(\xi, z_{m}(\xi), z_{m}^{\prime}(\xi)\right){ }^{\theta} R_{\xi_{k}}^{m}(\xi)\right\rangle_{L_{\rho}^{2}} \bar{\psi}_{j}^{m}(\xi) \\
& =\sum_{j=0}^{m-2} \sum_{k=0}^{j} \beta_{j k}^{m} g\left(\xi_{k}, z_{m}\left(\xi_{k}\right), z_{m}^{\prime}\left(\xi_{k}\right)\right) \bar{\psi}_{j}^{m}(\xi) .
\end{aligned}
$$

The proof is completed.

Theorem 7. If $z_{m} \in{ }^{\theta} \Omega_{\rho}^{m}[0,1]$, then $\left|z_{m}^{(s)}(\xi)\right| \leq F\left\|z_{m}\right\|_{\theta} \Omega_{\rho}^{m}$ for $s=0, \ldots, m-1$ and $m \geq 2$, where $F$ is a constant.

Proof. We have $z_{m}^{(s)}(\xi)=\left\langle z_{m}(\cdot), \partial_{\tilde{\xi}}^{s}{ }^{\theta} R_{\tilde{\xi}}^{m}(\cdot)\right\rangle_{\theta} \Omega_{\rho}^{m}$ for any $\xi, x \in[0,1], s=0, \ldots, m-1$. From the expression of ${ }^{\theta} R_{\xi}^{m}(x)$, it pursues that $\left\|\partial_{\xi}^{s \theta} R_{\xi}^{m}(x)\right\|_{{ }_{\theta} \Omega_{\rho}^{m}} \leq F_{s}, s=0, \ldots, m-1$. 
So,

$$
\begin{aligned}
\left|z_{m}^{(s)}(\xi)\right| & =\left|\left\langle z_{m}(\cdot), \partial_{\xi}^{s}{ }^{\theta} R_{\xi}^{m}(\cdot)\right\rangle_{\theta} \Omega_{\rho}^{m}\right| \\
& \leq\left\|z_{m}\right\|_{{ }^{\theta} \Omega_{\rho}^{m}[0,1]}\left\|\partial_{\tilde{\zeta}}^{s} R_{\tilde{\xi}}^{m}\right\|_{\theta} \Omega_{\rho}^{m} \\
& \leq F_{s}\left\|z_{m}\right\|_{{ }_{\theta} \Omega_{\rho}^{m}}, s=0, \ldots, m-1 .
\end{aligned}
$$

Therefore, $\left\|z_{m}^{(s)}\right\| \leq \max \left\{F_{0}, \ldots, F_{m-1}\right\}\left\|z_{m}\right\|_{{ }_{\theta} \Omega_{\rho}^{m}}, s=0, \ldots, m-1$.

Theorem 8. The approximate solution $z_{m}$ and its derivatives $z_{m}^{(s)}$, respectively, uniformly converge to the exact solution $z$ and its derivatives $z^{(s)}(s=0, \ldots, m-1)$.

Proof. By using Theorem 7 for any $\xi \in[0,1]$ we get

$$
\begin{aligned}
\left|z_{m}^{(s)}(\xi)-z^{(s)}(\xi)\right| & =\left|\left\langle z_{m}(\cdot)-z(\cdot), \partial_{\xi}^{s}{ }^{\theta} R_{\tilde{\xi}}^{m}(\cdot)\right\rangle\right|_{{ }_{\theta} \Omega_{\rho}^{m}} \\
& \leq\left\|\partial_{\xi}^{s}{ }^{\theta} R_{\xi}^{m}\right\|_{{ }_{\theta} \Omega_{\rho}^{m}}\left\|z_{m}-z\right\|_{{ }_{\theta} \Omega_{\rho}^{m}} \\
& \leq F_{s}\left\|z_{m}-z\right\|_{{ }_{\theta} \Omega_{\rho}^{m}}, s=0, \ldots, m-1 .
\end{aligned}
$$

where $F_{0}, \ldots, F_{m-1}$ are positive constants. Therefore, if $z_{m} \rightarrow z$ in the norm of ${ }^{\theta} \Omega_{\rho}^{m}[0,1]$ as $m \rightarrow \infty, z_{m}$ and its derivatives $z_{m}^{\prime}, \ldots, z_{m}^{(m-1)}$, respectively, uniformly converge to $z$ and its derivatives $z^{\prime}, \ldots, z^{(m-1)}$. This completes the proof.

If the considered problem is linear, a numerical solution can be obtained directly from (15). However, for a nonlinear problem, the following iterative procedure can be constructed.

\subsection{Construction of Iterative Procedure}

In this subsection, we will use the following iterative sequence to overcome the nonlinearity of the problem, $y_{m, n}(\xi)$, inserting,

$$
\left\{\begin{array}{l}
\operatorname{Lym,n}(\xi)=g\left(\xi, z_{m, n-1}(\xi), z_{m, n-1}^{\prime}(\xi)\right) \\
z_{m, n}(\xi)=P_{m-1} y_{m, n}(\xi)
\end{array}\right.
$$

here, an orthogonal projection operator is defined as $P_{m-1}:{ }^{\theta} \Omega_{\rho}^{m}[0,1] \rightarrow \operatorname{span}\left\{\bar{\psi}_{0}^{m}, \bar{\psi}_{1}^{m}, \ldots, \bar{\psi}_{m-2}^{m}\right\}$ and $y_{m, n}(\xi) \in{ }^{\theta} \Omega_{\rho}^{m}[0,1]$ shows the $n$-th iterative numerical solution of (16). Then, the following important theorem will be given for the iterative procedure.

Theorem 9. If $\left\{\xi_{j}\right\}_{j=0}^{m-2}$ are distinct points in open interval $(0,1)$ for $m \geq 2$, then

$$
y_{m, n}(\xi)=\sum_{j=0}^{m-2} \sum_{k=0}^{j} \beta_{j k}^{m} g\left(\xi_{k}, z_{m, n-1}\left(\xi_{k}\right), z_{m, n-1}^{\prime}\left(\xi_{k}\right)\right) \bar{\psi}_{j}^{m}(\xi)
$$


Proof. Since $y_{m, n}(\xi) \in{ }^{\theta} \Omega_{\rho}^{m}[0,1],\left\{\bar{\psi}_{j}^{m}(\xi)\right\}_{j=0}^{m-2}$ is the complete orthonormal system in ${ }^{\theta} \Omega_{\rho}^{m}[0,1]$,

$$
\begin{aligned}
y_{m, n}(\xi) & =\sum_{j=0}^{m-2}\left\langle y_{m, n}(\xi), \bar{\psi}_{j}^{m}(\xi)\right\rangle_{\theta} \Omega_{\rho}^{m} \bar{\psi}_{j}^{m}(\xi) \\
& =\sum_{j=0}^{m-2}\left\langle y_{m, n}(\xi), \sum_{k=0}^{j} \beta_{j k}^{m} \psi_{k}^{m}(\xi)\right\rangle_{\theta} \Omega_{\rho}^{m} \bar{\psi}_{j}^{m}(\xi) \\
& =\sum_{j=0}^{m-2} \sum_{k=0}^{j} \beta_{j k}^{m}\left\langle y_{m, n}(\xi), \psi_{k}^{m}(\xi)\right\rangle_{\theta} \Omega_{\rho}^{m} \bar{\psi}_{j}^{m}(\xi) \\
& =\sum_{j=0}^{m-2} \sum_{k=0}^{j} \beta_{j k}^{m}\left\langle y_{m, n}(\xi), L^{* \theta} R_{\tilde{\zeta}_{k}}^{m}(\xi)\right\rangle_{\theta} \Omega_{\rho}^{m} \bar{\psi}_{j}^{m}(\xi) \\
& =\sum_{j=0}^{m-2} \sum_{k=0}^{j} \beta_{j k}^{m}\left\langle L y_{m, n}(\xi),{ }^{\theta} R_{\xi_{k}}^{m}(\xi)\right\rangle_{L_{\rho}^{2}} \bar{\psi}_{j}^{m}(\xi) \\
& =\sum_{j=0}^{m-2} \sum_{k=0}^{j} \beta_{j k}^{m}\left\langle g\left(\xi, z_{m, n-1}(\xi), z_{m, n-1}^{\prime}(\xi)\right)^{\theta} R_{\xi_{k}}^{m}(\xi)\right\rangle_{L_{\rho}^{2}} \bar{\psi}_{j}^{m}(\xi) \\
& =\sum_{j=0}^{m-2} \sum_{k=0}^{j} \beta_{j k}^{m} g\left(\xi_{k}, z_{m, n-1}\left(\xi_{k}\right), z_{m, n-1}^{\prime}\left(\xi_{k}\right)\right) \bar{\psi}_{j}^{m}(\xi) .
\end{aligned}
$$

This completes the proof.

Taking $z_{m, 0}(\xi)=0$ and define the iterative sequence

$$
z_{m, n}(\xi)=P_{m-1} y_{m, n}(\xi)=\sum_{j=0}^{m-2} \sum_{k=0}^{j} \beta_{j k}^{m} g\left(\xi_{k}, z_{m, n-1}\left(\xi_{k}\right), z_{m, n-1}^{\prime}\left(\xi_{k}\right)\right) \bar{\psi}_{j}^{m}(\xi), n=1,2, \ldots
$$

Remark 1. For obtaining homogeneous boundary conditions in Equation (2), if the $z(\xi)=v(\xi)+a \xi^{2}+b \xi+c$ transformation is done, then coefficients can be found as $a=\left(\gamma_{2}-\gamma_{0}\right) /\left(1-\gamma_{1}\right), b=\theta\left(\gamma_{0}-\gamma_{2}\right) /\left(1-\gamma_{1}\right)$, $c=\gamma_{0}$. Here, $v(\xi)$ is a new unknown variable, and $v(0)=v(\theta)=v(1)=0$ homogeneous boundary conditions are also satisfied.

\section{Numerical Applications}

In this section, some nonlinear three-point boundary value problems are considered to exemplify the accuracy and efficiency of the proposed approach. Numerical results, which are achieved by L-RKM, are shown with tables.

Example 1. We consider the following multi-order fractional nonlinear three-point boundary value problem with a Caputo derivative:

$$
\begin{gathered}
{ }^{c} D^{\alpha} z(\xi)+(\xi+1){ }^{c} D^{\beta} z(\xi)+\xi z(\xi)-z^{2}(\xi)=f(\xi), \quad 1<\alpha \leq 2 . \quad 0<\beta \leq 1 . \\
z(0)=z\left(\frac{1}{2}\right)=z(1)=0 .
\end{gathered}
$$

here, $f(\xi)$ is a known function such that the exact solution of this problem is $z(\xi)=\xi\left(\xi-\frac{1}{2}\right)(\xi-1)$. 
By using the proposed approach for Equations (19) and (20), and choosing nodal points as $\xi_{j}=\frac{j+0.3}{m}, j=$ $0,1,2, \ldots, m-2$, the approximate solution $z_{m, n}(\xi)$ is computed by Equation (18). For Equations (19) and (20), a comparison of absolute errors for different $\alpha, \beta$ values are demonstrated in Tables 1 and 2 and a comparison of the exact solution and numerical solution for $\alpha=1.75$ and $\beta=0.75$ is given in Table 3 .

Table 1. Comparison of absolute error of Example 1 for various $\alpha, \beta(m=3, n=3)$.

\begin{tabular}{|c|c|c|c|c|c|}
\hline$x$ & $\alpha=2, \beta=1$ & $\alpha=1.9, \beta=0.9$ & $\alpha=1.8, \beta=0.8$ & $\alpha=1.7, \beta=0.7$ & $\alpha=1.6, \beta=0.6$ \\
\hline 0 & 0 & 0 & 0 & 0 & 0 \\
\hline 0.1 & $3.25 \times 10^{-11}$ & $6.08 \times 10^{-11}$ & $3.70 \times 10^{-12}$ & $4.45 \times 10^{-10}$ & $3.14 \times 10^{-9}$ \\
\hline 0.2 & $5.45 \times 10^{-11}$ & $8.75 \times 10^{-11}$ & $4.93 \times 10^{-12}$ & $8.70 \times 10^{-10}$ & $5.51 \times 10^{-9}$ \\
\hline 0.3 & $6.97 \times 10^{-11}$ & $9.00 \times 10^{-11}$ & $4.34 \times 10^{-12}$ & $1.32 \times 10^{-9}$ & $7.36 \times 10^{-9}$ \\
\hline 0.4 & $8.17 \times 10^{-11}$ & $7.81 \times 10^{-11}$ & $2.55 \times 10^{-12}$ & $1.85 \times 10^{-9}$ & $8.96 \times 10^{-9}$ \\
\hline 0.5 & 0 & 0 & 0 & 0 & 0 \\
\hline 0.6 & $1.10 \times 10^{-10}$ & $5.14 \times 10^{-11}$ & $2.03 \times 10^{-12}$ & $3.36 \times 10^{-9}$ & $1.24 \times 10^{-8}$ \\
\hline 0.7 & $1.34 \times 10^{-10}$ & $5.65 \times 10^{-11}$ & $3.56 \times 10^{-12}$ & $4.44 \times 10^{-9}$ & $1.49 \times 10^{-8}$ \\
\hline 0.8 & $1.70 \times 10^{-10}$ & $8.70 \times 10^{-11}$ & $3.73 \times 10^{-12}$ & $5.80 \times 10^{-9}$ & $1.81 \times 10^{-8}$ \\
\hline 0.9 & $2.21 \times 10^{-10}$ & $1.53 \times 10^{-11}$ & $1.89 \times 10^{-12}$ & $7.49 \times 10^{-9}$ & $2.25 \times 10^{-8}$ \\
\hline 1 & 0 & 0 & 0 & 0 & 0 \\
\hline
\end{tabular}

Table 2. Comparison of absolute error of Example 1 for various $\alpha, \beta(m=3, n=5)$.

\begin{tabular}{|c|c|c|c|c|c|}
\hline$x$ & $\alpha=2, \beta=1$ & $\alpha=1.9, \beta=0.9$ & $\alpha=1.8, \beta=0.8$ & $\alpha=1.7, \beta=0.7$ & $\alpha=1.6, \beta=0.6$ \\
\hline 0 & 0 & 0 & 0 & 0 & 0 \\
\hline 0.1 & $3.78 \times 10^{-17}$ & $1.33 \times 10^{-16}$ & $2.49 \times 10^{-19}$ & $4.86 \times 10^{-15}$ & $7.48 \times 10^{-14}$ \\
\hline 0.2 & $5.27 \times 10^{-17}$ & $1.94 \times 10^{-16}$ & $3.36 \times 10^{-19}$ & $1.13 \times 10^{-14}$ & $1.36 \times 10^{-13}$ \\
\hline 0.3 & $5.10 \times 10^{-17}$ & $2.03 \times 10^{-16}$ & $3.80 \times 10^{-19}$ & $1.99 \times 10^{-14}$ & $1.91 \times 10^{-13}$ \\
\hline 0.4 & $3.91 \times 10^{-17}$ & $1.81 \times 10^{-16}$ & $5.20 \times 10^{-19}$ & $3.09 \times 10^{-14}$ & $2.43 \times 10^{-13}$ \\
\hline 0.5 & 0 & 0 & 0 & 0 & 0 \\
\hline 0.6 & $1.05 \times 10^{-17}$ & $1.36 \times 10^{-16}$ & $1.56 \times 10^{-18}$ & $6.18 \times 10^{-14}$ & $3.60 \times 10^{-13}$ \\
\hline 0.7 & $6.65 \times 10^{-18}$ & $1.57 \times 10^{-16}$ & $2.74 \times 10^{-18}$ & $8.25 \times 10^{-14}$ & $4.36 \times 10^{-13}$ \\
\hline 0.8 & $1.81 \times 10^{-17}$ & $2.34 \times 10^{-16}$ & $4.50 \times 10^{-18}$ & $1.07 \times 10^{-14}$ & $5.30 \times 10^{-13}$ \\
\hline 0.9 & $5.14 \times 10^{-17}$ & $3.91 \times 10^{-16}$ & $7.02 \times 10^{-18}$ & $1.36 \times 10^{-14}$ & $6.48 \times 10^{-13}$ \\
\hline 1 & 0 & 0 & 0 & 0 & 0 \\
\hline
\end{tabular}

Table 3. Numerical results of Example 1 for $m=5, n=9$ values $(\alpha=1.75, \beta=0.75)$.

\begin{tabular}{cccc}
\hline$x$ & Exact Sol. & Approximate Sol. & Absolute Error \\
\hline 0.0 & 0.000000000000000000000 & 0.000000000000000000000 & 0 \\
0.1 & 0.036000000000000000000 & 0.036000000000000000018 & $1.80 \times 10^{-20}$ \\
0.2 & 0.048000000000000000000 & 0.048000000000000000044 & $4.40 \times 10^{-20}$ \\
0.3 & 0.042000000000000000000 & 0.042000000000000000061 & $6.10 \times 10^{-20}$ \\
0.4 & 0.024000000000000000000 & 0.024000000000000000071 & $7.10 \times 10^{-20}$ \\
0.5 & 0.000000000000000000000 & 0.000000000000000000000 & 0 \\
0.6 & -0.024000000000000000000 & -0.023999999999999999899 & $1.01 \times 10^{-19}$ \\
0.7 & -0.042000000000000000000 & -0.041999999999999999819 & $1.81 \times 10^{-19}$ \\
0.8 & -0.048000000000000000000 & -0.047999999999999999694 & $3.06 \times 10^{-19}$ \\
0.9 & -0.036000000000000000000 & -0.035999999999999999491 & $5.09 \times 10^{-19}$ \\
1 & 0.000000000000000000000 & 0.000000000000000000000 & 0 \\
\hline
\end{tabular}


Example 2. We take care of the following multi-order fractional nonlinear three-point boundary value problem with a Caputo derivative

$$
\begin{gathered}
\xi^{2}{ }^{c} D^{\alpha} z(\xi)+\left(\xi^{2}-1\right){ }^{c} D^{\beta} z(\xi)+\xi^{3} z(\xi)-z(\xi) z^{\prime}(\xi)-z^{3}(\xi)=f(\xi), \quad 1<\alpha \leq 2 . \quad 0<\beta \leq 1 . \\
z(0)=z\left(\frac{3}{5}\right)=z(1)=0 .
\end{gathered}
$$

Here, $f(\xi)$ is a known function such that the exact solution of this problem is $z(\xi)=\xi\left(\xi-\frac{3}{5}\right)(\xi-1)$.

By using the proposed approach for Equations (21) and (22), and choosing the nodal points as $\xi_{j}=\frac{j+0.3}{m}, j=$ $0,1,2, \ldots, m-2$, the approximate solution $z_{m, n}(\xi)$ is computed by Equation (18). For (21) and (22), a comparison of absolute errors for different $\alpha, \beta$ values are demonstrated in Tables 4 and 5 and a comparison of the exact solution and numerical solution for $\alpha=1.75$ and $\beta=0.75$ is given in Table 6 .

Table 4. Comparison of absolute error of Example 2 for various $\alpha, \beta(m=3, n=8)$.

\begin{tabular}{cccccc}
\hline $\boldsymbol{x}$ & $\boldsymbol{\alpha}=\mathbf{2 , \boldsymbol { \beta } = \mathbf { 1 }}$ & $\boldsymbol{\alpha}=\mathbf{1 . 9} \boldsymbol{\beta} \boldsymbol{\beta}=\mathbf{0 . 9}$ & $\boldsymbol{\alpha}=\mathbf{1 . 8} \boldsymbol{\beta}=\mathbf{0 . 8}$ & $\boldsymbol{\alpha}=\mathbf{1 . 7 ,} \boldsymbol{\beta}=\mathbf{0 . 7}$ & $\boldsymbol{\alpha}=\mathbf{1 . 6} \boldsymbol{\beta}=\mathbf{0 . 6}$ \\
\hline 0 & 0 & 0 & 0 & 0 & 0 \\
0.1 & $5.00 \times 10^{-11}$ & $4.11 \times 10^{-15}$ & $2.78 \times 10^{-13}$ & $6.10 \times 10^{-12}$ & $1.40 \times 10^{-12}$ \\
0.2 & $8.39 \times 10^{-11}$ & $3.45 \times 10^{-15}$ & $1.87 \times 10^{-13}$ & $1.17 \times 10^{-11}$ & $2.42 \times 10^{-11}$ \\
0.3 & $1.06 \times 10^{-10}$ & $1.24 \times 10^{-15}$ & $1.87 \times 10^{-13}$ & $1.45 \times 10^{-11}$ & $7.08 \times 10^{-11}$ \\
0.4 & $1.23 \times 10^{-10}$ & $9.21 \times 10^{-15}$ & $7.60 \times 10^{-13}$ & $1.20 \times 10^{-11}$ & $1.32 \times 10^{-10}$ \\
0.5 & $1.39 \times 10^{-10}$ & $1.97 \times 10^{-14}$ & $1.44 \times 10^{-12}$ & $1.75 \times 10^{-12}$ & $2.02 \times 10^{-10}$ \\
0.6 & 0 & 0 & 0 & 0 & 0 \\
0.7 & $1.88 \times 10^{-10}$ & $4.52 \times 10^{-14}$ & $2.81 \times 10^{-12}$ & $5.17 \times 10^{-11}$ & $3.45 \times 10^{-10}$ \\
0.8 & $2.31 \times 10^{-10}$ & $5.88 \times 10^{-14}$ & $3.32 \times 10^{-12}$ & $9.98 \times 10^{-11}$ & $4.05 \times 10^{-10}$ \\
0.9 & $2.93 \times 10^{-10}$ & $7.18 \times 10^{-14}$ & $3.60 \times 10^{-12}$ & $1.65 \times 10^{-10}$ & $4.49 \times 10^{-10}$ \\
1 & 0 & 0 & 0 & 0 & 0 \\
\hline
\end{tabular}

Table 5. Comparison of absolute error of Example 2 for various $\alpha, \beta(m=3, n=10)$.

\begin{tabular}{cccccc}
\hline $\boldsymbol{x}$ & $\boldsymbol{\alpha}=\mathbf{2}, \boldsymbol{\beta}=\mathbf{1}$ & $\boldsymbol{\alpha}=\mathbf{1 . 9} \boldsymbol{\beta} \mathbf{\beta}=\mathbf{0 . 9}$ & $\boldsymbol{\alpha}=\mathbf{1 . 8 ,} \boldsymbol{\beta}=\mathbf{0 . 8}$ & $\boldsymbol{\alpha}=\mathbf{1 . 7 ,} \boldsymbol{\beta}=\mathbf{0 . 7}$ & $\boldsymbol{\alpha}=\mathbf{1 . 6 ,} \boldsymbol{\beta}=\mathbf{0 . 6}$ \\
\hline 0 & 0 & 0 & 0 & 0 & 0 \\
0.1 & $3.49 \times 10^{-13}$ & $1.93 \times 10^{-18}$ & $4.48 \times 10^{-16}$ & $2.68 \times 10^{-14}$ & $4.75 \times 10^{-15}$ \\
0.2 & $5.87 \times 10^{-13}$ & $8.32 \times 10^{-19}$ & $2.99 \times 10^{-16}$ & $5.58 \times 10^{-14}$ & $7.68 \times 10^{-14}$ \\
0.3 & $7.47 \times 10^{-13}$ & $2.90 \times 10^{-18}$ & $3.06 \times 10^{-16}$ & $7.18 \times 10^{-14}$ & $2.25 \times 10^{-13}$ \\
0.4 & $8.65 \times 10^{-13}$ & $8.81 \times 10^{-18}$ & $1.23 \times 10^{-15}$ & $6.00 \times 10^{-14}$ & $4.21 \times 10^{-13}$ \\
0.5 & $9.76 \times 10^{-13}$ & $1.65 \times 10^{-17}$ & $2.34 \times 10^{-15}$ & $5.54 \times 10^{-15}$ & $6.45 \times 10^{-13}$ \\
0.6 & 0 & 0 & 0 & 0 & 0 \\
0.7 & $1.31 \times 10^{-12}$ & $3.55 \times 10^{-17}$ & $4.55 \times 10^{-15}$ & $2.91 \times 10^{-13}$ & $1.09 \times 10^{-12}$ \\
0.8 & $1.61 \times 10^{-12}$ & $4.60 \times 10^{-17}$ & $5.37 \times 10^{-15}$ & $5.64 \times 10^{-13}$ & $1.29 \times 10^{-12}$ \\
0.9 & $2.05 \times 10^{-12}$ & $5.65 \times 10^{-17}$ & $5.83 \times 10^{-15}$ & $9.39 \times 10^{-13}$ & $1.43 \times 10^{-12}$ \\
1 & 0 & 0 & 0 & 0 & 0 \\
\hline
\end{tabular}


Table 6. Numerical results of Example 2 for $m=5, n=9$ values $(\alpha=1.75, \beta=0.75)$.

\begin{tabular}{cccc}
\hline$x$ & Exact Sol. & Approximate Sol. & Absolute Error \\
\hline 0.0 & 0.000000000000000000000 & 0.000000000000000000000 & 0 \\
0.1 & 0.045000000000000000000 & 0.045000000000480782793 & $4.80 \times 10^{-13}$ \\
0.2 & 0.064000000000000000000 & 0.064000000000580045412 & $5.80 \times 10^{-13}$ \\
0.3 & 0.063000000000000000000 & 0.063000000000488602930 & $4.88 \times 10^{-13}$ \\
0.4 & 0.048000000000000000000 & 0.048000000000398645450 & $3.98 \times 10^{-13}$ \\
0.5 & 0.025000000000000000000 & 0.025000000000468872800 & $4.68 \times 10^{-13}$ \\
0.6 & 0.000000000000000000000 & 0.000000000000000000000 & 0 \\
0.7 & -0.021000000000000000000 & -0.020999999998651962040 & $1.34 \times 10^{-12}$ \\
0.8 & -0.032000000000000000000 & -0.031999999998006864020 & $1.99 \times 10^{-12}$ \\
0.9 & -0.027000000000000000000 & -0.026999999997598991320 & $2.40 \times 10^{-12}$ \\
1 & 0.000000000000000000000 & 0.000000000000000000000 & 0 \\
\hline
\end{tabular}

\section{Conclusions}

In this research, a novel numerical approach called L-RKM has been proposed and successfully implemented to find the approximate solution of a multi-order fractional nonlinear three-point boundary value problem with a Caputo derivative. For nonlinear problems, a new iterative process is proposed. Numerical findings show that the present approach is efficient and convenient for solving three-point boundary value problems with fractional order.

Author Contributions: Writing-original draft, M.G.S.; writing-review \& editing, O.S. Both authors have read and agreed to the published version of the manuscript.

Funding: This research received no external funding.

Conflicts of Interest: The authors declare no conflict of interest.

\section{References}

1. Lin, Y.; Niu, J.; Cui, M. A numerical solution to nonlinear second order three-point boundary value problems in the reproducing kernel space. Appl. Math. Comput. 2012, 218, 7362-7368. [CrossRef]

2. Rehman, M.; Khan, R.A.; Asif, N.A. Three point boundary value problems for nonlinear fractional differential equations. Acta Math. 2011, 31, 1337-1346. [CrossRef]

3. Geng, F. Solving singular second order three-point boundary value problems using reproducing kernel Hilbert space method. Appl. Math. Comput. 2009 215, 2095-2102. [CrossRef]

4. Zhang, C.P.; Niu, J.; Lin, Y.Z. Numerical solutions for the three-point boundary value problem of nonlinear fractional differential equations. Abstr. Appl. Anal. 2012, 2012,1-16. [CrossRef]

5. Etemad, S.; Ntouyas, S.K.; Tariboon, J.Existence results for three-point boundary value problems for nonlinear fractional differential equations. J. Nonlinear Sci. Appl. 2016, 9, 2105-2116. [CrossRef]

6. Wu, B.; Li, X. Application of reproducing kernel method to third order three-point boundary value problems. Appl. Math. Comput. 2010, 217, 3425-3428. [CrossRef]

7. Ahmad, B.; Alghanmi, M.; Ntouyas, S.K.; Alsaedi, A. A study of fractional differential equations and inclusions involving generalized Caputo-type derivative equipped with generalized fractional integral boundary conditions. AIMS Math. 2018, 4, 26-42. [CrossRef]

8. Podlubny, I. Fractional Differential Equations; Academic Press: New York, NY, USA, 1999.

9. Lakshmikantham, V.; Leela, S.; Vasundhara Devi, J. Theory of Fractional Dynamic Systems; Cambridge Scientific Publishers: Cambridge, UK, 2009.

10. Hilfer, R. Applications of Fractional Calculus in Physics; World Scientific: Singapore, 2000.

11. Tarasov, V.E. Fractional Dynamics: Application of Fractional Calculus to Dynamics of Particles, Fields and Media; Springer: Berlin, Germany, 2010. 
12. Diethelm, K. The Analysis of Fractional Differential Equations. Lecture Notes in Mathematics; Springer-Verlag: Berlin/Heidelberg, Germany, 2010.

13. Kilbas, A.A.; Srivastava, H.M.; Trujillo, J.J. Theory and Applications of Fractional Differential Equations; B.V, Elsevier Science: Amsterdam, The Netherlands, 2006.

14. Khalouta, A.; Kadem, A. A new numerical technique for solving Caputo time-fractional biological population equation. AIMS Math. 2019, 4, 1307-1319. [CrossRef]

15. Tadjeran, C.; Meerschaert, M.M. A second-order accurate numerical method for the two-dimensional fractional diffusion equation. J. Comput. Phys. 2007, 220, 813-823. [CrossRef]

16. Esen, A.; Tasbozan, O. Numerical solution of time fractional Burgers equation by cubic B-spline finite elements. Mediterr. J. Math. 2016, 13, 1325-1337. [CrossRef]

17. Sakar, M.G.; Uludag, F.; Erdogan, F. Numerical solution of time-fractional nonlinear PDEs with proportional delays by homotopy perturbation method. Appl. Math. Model. 2016, 40, 6639-6649. [CrossRef]

18. Saeed, U.; Rehman, M. Haar wavelet-quasilinearization technique for fractional nonlinear differential equations. Appl. Math. Comput. 2013, 220, 630-648. [CrossRef]

19. Pezza, L.; Pitolli, F. A multiscale collocation method for fractional differential problems. Math. Comput. Simul. 2018, 147, 210-219. [CrossRef]

20. Sakar, M.G.; Erdogan, F. The homotopy analysis method for solving the time-fractional Fornberg-Whitham equation and comparison with Adomian's decomposition method. Appl. Math. Model. 2013, 37, 1634-1641. [CrossRef]

21. Jafari, H.; Jassim, H.K.; Moshokoa, S.P.; Ariyan, V.M.; Tchier, F. Reduced differential transform method for partial differential equations within local fractional derivative operators. Adv. Mech. Eng. 2016, 4, 1-6. [CrossRef]

22. Sakar, M.G.; Saldır, O. Improving variational iteration method with auxiliary parameter for nonlinear time-fractional partial differential equations. J. Optim. Theory Appl. 2017, 174, 530-549. [CrossRef]

23. Xu, M.Q.; Lin, Y.Z. Simplified reproducing kernel method for fractional differential equations with delay. Appl. Math. Lett. 2016, 52, 156-161. [CrossRef]

24. Wang, Y.L.; Du, M.J.; Temuer, C.L.; Tian, D. Using reproducing kernel for solving a class of time-fractional telegraph equation with initial value conditions. Int. J. Comput. Math. 2018, 95, 1609-1621. [CrossRef]

25. Kadem, A.; Baleanu, D. Fractional radiative transfer equation within Chebyshev spectral approach. Comput. Math. Appl. 2010, 59, 1865-1873. [CrossRef]

26. Eldien, S.S.E.; Hafez, R.M.; Bhrawy, A.H.; Baleanu, D.; Kalaawy, A.A.E. New numerical approach for fractional variational problems using shifted Legendre orthonormal polynomials. J. Optim. Theory Appl. 2017, 174, $295-320$. [CrossRef]

27. Sakar, M.G.; Akgül, A.; Baleanu, D. On solutions of fractional Riccati differential equations. Adv. Differ. Equ. 2017, 39, 1-10. [CrossRef]

28. Sakar, M.G.; Saldır, O.; Erdogan, F. A hybrid method for singularly perturbed convection-diffusion equation. Int. J. Appl. Comput. 2019, 5, 1-17. [CrossRef]

29. Erturk, V.S.; Momani, S.; Odibat, Z. Application of generalized differential transform method to multi-order fractional differential equations. Commun. Nonlinear Sci. Numer. Simul. 2008, 13, 1642-1654. [CrossRef]

30. Dabiri, A.; Butcher, E.A. Stable fractional Chebyshev differentiation matrix for the numerical solution of multi-order fractional differential equations. Nonlinear Dyn. 2017, 90, 185-201. [CrossRef]

31. Sakar, M.G.; Saldır, O; Akgül, A. A novel technique for fractional Bagley-Torvik equation. Proc. Natl. Acad. Sci., India Sect. A Phys. Sci. 2019, 89, 539-545. [CrossRef]

32. Khalegi, M.; Babolian, E.; Abbasbandy, S. Chebyshev reproducing kernel method: application to two-point boundary value problems. Adv. Differ. Equ. 2017, 26, 1-19.

33. Zaremba, S.Sur le Calcul Numérique des Fonctions Demandées Dans le probléme de Dirichlet et le Problème Hydrodynamique. Bulletin International de l'Académie des Sciences de Cracovie 1908, 125-195.

34. Cui, M.; Lin, Y. Nonlinear Numerical Analysis in the Reproducing Kernel Space; Nova Science: New York, NY, USA, 2009.

35. Alpay, D. Reproducing Kernel Spaces and Applications; Springer: Berlin/Heidelberg, Germany, 2003. 
36. Geng, F.; Cui, M. A reproducing kernel method for solving nonlocal fractional boundary value problems. Appl. Math. Lett. 2012, 25, 818-823. [CrossRef]

37. Sakar, M.G. Iterative reproducing kernel Hilbert spaces method for Riccati differential equation. J. Comput. Appl. Math. 2017, 309, 163-174. [CrossRef]

38. Geng, F.; Cui, M. New method based on the HPM and RKHSM for solving forced Duffing equations with integral boundary conditions. J. Comput. Appl. Math. 2009, 233, 165-172. [CrossRef]

39. Saldır, O.; Sakar, M.G.; Erdogan, F. Numerical solution of fractional order Burgers' equation with Dirichlet and Neumann boundary conditions by reproducing kernel method. Fractal Fract. 2020, 4, 27. [CrossRef]

40. Saldır, O.; Sakar, M.G.; Erdogan, F. Numerical solution of time-fractional Kawahara equation using reproducing kernel method with error estimate. Comput. Appl. Math. 2019, 38, 1-23. [CrossRef]

41. Sakar, M.G.; Saldır, O. A novel iterative solution for time-fractional Boussinesq equation by reproducing kernel method. J. Appl. Math. Comput. 2020, 64, 227-254. [CrossRef]

42. Jiang, W.; Tian, T. Numerical solution of nonlinear Volterra integro-differential equations of fractional order by the reproducing kernel method. Appl. Math. Model. 2015, 39, 4871-4876. [CrossRef]

43. Sakar, M.G.; Saldır, O.; Akgül, A. Numerical solution of fractional Bratu type equations with Legendre reproducing kernel method. Int. J. Appl. Comput. Math. 2018, 126, 1-14. [CrossRef]

44. Kaplan, W. Advanced Calculus (5E); Pearson Education: New York, NY, USA, 2002.

45. Rainville, E.D. Special Functions; Chelsea Publishing Co.: New York, NY, USA, 1960.

46. Szegö, G. Orthogonal Polynomials; American Mathematical Society Colloquium Publications: Providence, RI, USA, 1939.

47. Aronszajn, N. Theory of reproducing kernels. Trans. Am. Math. Soc. 1950, 68, 337-404. [CrossRef]

Publisher's Note: MDPI stays neutral with regard to jurisdictional claims in published maps and institutional affiliations.

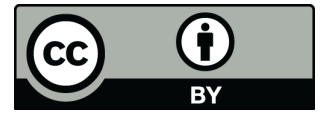

(C) 2020 by the authors. Licensee MDPI, Basel, Switzerland. This article is an open access article distributed under the terms and conditions of the Creative Commons Attribution (CC BY) license (http:/ / creativecommons.org/licenses/by/4.0/). 